Fístula ureteroiliaca como complicación de colocación de catéter JJ

\title{
Ureteroiliac fistula as a JJ catheter placement complication
}




\section{00308}

Fístula ureteroiliaca como complicación de colocación de catéter JJ

Ureteroiliac fistula as a J catheter placement complication

Amaia Ormaechevarria Iturrizaga, Juan Carlos Fernández Fernández, Melina Vega de Ceniga, June Blanco Larizgoitia, Luis Estallo Laliena Servicio de Angiología, Cirugía Vascular y Endovascular. Hospital Universitario de Galdakao-Usansolo. Galdakao, Bizkaia

Recibido: 07/04/2021

Aceptado: 21/04/2021

Correspondencia: Amaia Ormaechevarria Iturrizaga. Servicio de Angiología, Cirugía Vascular y Endovascular. Hospital Universitario de Galdakao-Usansolo. Labeaga Auzoa. 48960 Galdakao, Bizkaia email: amaia.ormaechevarria@gmail.com

DOI: 10.20960/angiologia.00308

Conflicto de interés: los autores declaran no tener ningún conflicto de interés.

\section{RESUMEN}

Introducción: la colocación de un catéter JJ es una maniobra urológica habitual. Pese a su relativa sencillez, esta técnica puede asociar complicaciones.

Caso clínico: se presenta un caso de fístula ureteroiliaca, una de las complicaciones más infrecuentes. Se trata de una mujer de 69 años con antecedentes de carcinoma de cérvix tratado con cirugía y radioterapia pélvica, atrofia obstructiva del riñón izquierdo, y atrapamiento ureteral derecho, que precisaba recambios periódicos 
de su catéter ureteral derecho. Durante el último recambio se objetivó sangrado activo y hematuria procedente del uréter derecho. Tras estudio mediante TAC, ureterorrenoscopia y arteriografía se diagnosticó una fístula ureteroiliaca derecha. Mediante punción percutánea ecoguiada femoral derecha, se implantó un stent recubierto de $8 \times 57 \mathrm{~mm}$ (Begraft ${ }^{\circledR}$ ) en la arteria iliaca externa derecha, con corrección instantánea del sangrado.

Discusión: la fístula ureteroiliaca es una complicación inusual tras la colocación de un catéter JJ. Se ha realizado una revisión de casos publicados sobre esta patología y se observan antecedentes de historia quirúrgica vascular y/u oncológica y tratamiento radioterápico pélvico previo en todos ellos. Tras esta revisión se concluye que el tratamiento endovascular es de primera elección, pues se trata de un abordaje mínimamente invasivo que ofrece opciones de diagnóstico y tratamiento de manera rápida y eficaz.

Palabras clave: Fístula ureteroiliaca. Complicación catéter JJ. Endoprótesis iliaca.

\section{ABSTRACT}

Introduction: the placement of a JJ catheter is a common urological maneuver. Despite its relative simplicity, this technique can have complications associated.

Case report: we present a case of a 69-year-old woman who developed a ureteroiliac fistula, one of the rarest complications after the placement of a JJ catheter. She had a history of cervix carcinoma treated with surgery and pelvic radiotherapy, with only one functional kidney due to obstructive atrophy of the left kidney, and right ureteral obstruction, and she required periodic replacement of her right ureteral catheter. During the last replacement, active bleeding and hematuria coming from the right ureter was observed; the study was completed with CT, ureterorenoscopy and arteriography, diagnosing a right ureteroiliac fistula. Using ultrasound-guided right femoral 
percutaneous puncture, an $8 \times 57 \mathrm{~mm}$ coated stent (Begraft $\AA$ ) was implanted in the right proximal external iliac artery, with instant correction of the bleeding.

Discussion: ureteroiliac fistula is an unusual complication after the placement of a JJ catheter. We performed a review of published cases and history of pelvic surgery and/or radiotherapy was described in all of them. We can conclude that endovascular treatment is currently the first-line management of ureteroiliac fistula. It is a minimally invasive approach that offers fast and effective diagnostic and treatment options.

Keywords: Ureteroiliac fistula. JJ catheter complication. Iliac endoprosthesis.

\section{INTRODUCCIÓN}

La colocación de un catéter JJ es una maniobra urológica habitual. En la actualidad presenta una indicación profiláctica asociada a procedimientos endourológicos para disminuir el riesgo de obstrucción por edema ureteral, coágulos o restos litiásicos; o terapéutica, como tratamiento de múltiples patologías, para permitir el drenaje correcto de la vía urinaria (1).

Pese a su relativa sencillez, esta técnica puede asociar complicaciones como la disuria o hematuria autolimitadas, migración del catéter, calcificación, rotura u obstrucción del mismo (1).

Presentamos un caso de fístula ureteroiliaca, una de las complicaciones más infrecuentes tras la colocación de un catéter JJ.

\section{CASO CLÍNICO}

Se trata de una mujer de 69 años con antecedente de carcinoma de cérvix tratado con cirugía (histerectomía + doble anexectomía + linfadenectomía pélvica y paraaórtica bilateral) + radioterapia pélvica adyuvante. Presentaba atrofia obstructiva del riñón izquierdo por 
cambios actínicos pélvicos y atrapamiento ureteral derecho como secuela del tratamiento. La paciente, monorrena funcional, se encontraba en seguimiento por el servicio de Nefrología y portaba un catéter ureteral derecho realizando recambios periódicos del mismo. Acude para un nuevo recambio y durante la intervención se objetiva sangrado, un coágulo vesical y hematuria procedente del uréter derecho por lo que se coloca un nuevo catéter JJ como drenaje. Tras la intervención se produce un deterioro agudo de la función renal con ascenso de las cifras de creatinina hasta $6 \mathrm{mg} / \mathrm{dL}$, respecto de su basal (2,5-3 mg/dL). Se solicita estudio con TAC donde se objetiva un urinoma a nivel de vías urinarias, requiriendo una nefrostomía percutánea evacuadora.

Cinco días más tarde la paciente desarrolla un sangrado a través de la nefrostomía. En una nueva TAC se evidencian un hematoma subcapsular y restos hemáticos intrarrenales. Posteriormente el estudio se complementa con una arteriografía donde se visualizan y embolizan dos pseudoaneurismas renales capsulares.

Los días posteriores presenta nuevamente hematuria anemizante alcanzando niveles de $\mathrm{Hb}$ de 7,2 g/dL; y mediante ureterorrenoscopia y arteriografía se diagnostica una fístula ureteroiliaca derecha (Fig. $1 \mathrm{~A}-\mathrm{C})$.

Bajo anestesia local y mediante punción percutánea ecoguiada femoral derecha, se implanta un stent recubierto de $8 \times 57 \mathrm{~mm}$ (Begraft ${ }^{\circledR}$ ) en la arteria iliaca externa proximal derecha (Fig. 1D), con corrección del sangrado. Posteriormente se retira el catéter J y se coloca una nefrostomía con balón permanente.

El posoperatorio es prolongado con recuperación de la función renal y resolución de la hematuria. Es dada de alta con doble antiagregación (ácido acetilsalicílico $100 \mathrm{mg}$ y clopidogrel $75 \mathrm{mg}$ ) durante 3 meses y profilaxis antibiótica ante cualquier procedimiento invasivo diagnóstico y/o terapéutico.

En los siguientes meses la paciente sufre deterioro progresivo de la función renal; se encuentra actualmente en prediálisis, sin nuevos 
episodios de hematuria. El seguimiento posterior en consulta muestra el stent iliaco permeable y sin complicaciones en controles ecográficos; la paciente se mantiene antiagregada con monoterapia, se encuentra asintomática desde el punto de vista vascular y conserva pulsos distales.

\section{DISCUSIÓN}

La fístula ureteroiliaca es una de las complicaciones más infrecuentes tras la colocación de un catéter JJ. Es importante la sospecha clínica inicial tras episodios reiterados de hematuria para un correcto diagnóstico ya que se trata de una complicación grave y potencialmente mortal.

Se ha realizado una revisión de casos similares que se recogen en la tabla I.

Cabe destacar los antecedentes oncológicos, cirugías y tratamiento radioterápico pélvico que se da en todos los casos registrados (3-13). Estos datos pueden hacer sospechar de una posible fístula y ayudar en el diagnóstico precoz y tratamiento primario de la lesión.

El diagnóstico de este tipo de patología no siempre se realiza de manera precoz. Por un lado, se trata de una patología poco frecuente y presenta una expresión clínica variada e intermitente. Puede presentarse como episodios de hematuria aislados, o presentar de manera aguda un sangrado importante, en caso de fístulas de alto 
inicial, con únicamente un caso (8) en el que se tuvo que reintervenir por persistencia de la fístula corrigiéndose en esta segunda cirugía.

En cuanto a la complicarinnoc a laron nlazo co oheorvan doe

caso de isquemia cricica y posterior amputacion supraconallea (b) y dos casos de fallecimiento, por complicación infecciosa (9) y por comorbilidad asociada a su ERC previa (9). Un paciente se perdió en

el seguimiento posterior (9). Cabe destacar un caso (7) con diagnóstico a los dos años de fístula uretero-arterio-entérica que requirió una laparotomía y cirugía multidisciplinar con resección de componentes fistulosos.

El tratamiento endovascular permite realizar un diagnóstico definitivo intraoperatorio en caso de alta sospecha y pruebas complementarias previas normales.

Esta técnica ofrece ventajas evidentes de acceso a distancia a la fístula ureteroiliaca evitando el campo quirúrgico hostil (por cirugías o radioterapia previa) y con opción de tratar el eje iliaco en toda su longitud según la complicación de la lesión. Además, es un procedimiento rápido y técnicamente sencillo.

Tras esta revisión concluimos que se debe mantener un alto nivel de sospecha de fístula ureteroiliaca en caso de hematuria persistente intermitente, en un paciente con cirugía oncológica o tratamiento radioterápico pélvico previo y que el procedimiento endovascular es de elección para el tratamiento de una fístula ureteroiliaca, pues se trata de un abordaje mínimamente invasivo que ofrece opciones de diagnóstico y tratamiento de manera rápida y eficaz.

\section{BIBLIOGRAFÍA}

1. Cepeda M, Mainez JA, de la Cruz B, Amón JH. Indicaciones y morbilidad asociada a los catéteres doble J. Arch Esp Urol 2016;69:462-70. 
2. Van den Bergh RC, Moll FL, de Vries JP, Lock TM. Arterioureteral fistulas: unusual uspects-systematic review of 139 cases. Urology 2009;74:251-5.

3. Mujo T, Priddy E, Harris JJ, Poulos E, Samman M. Unique Presentation of Hematuria in a Patient with Arterioureteral Fistula. Case Rep Radiol 2016;8682040.

4. Meester DJ, van Muiswinkel KW, Ameye F, Fransen H, Stockx L, Merckx $L$, et al. Endovascular treatment of a ureteroiliac fistula associated with ureteral double J-stenting and an aorticbifemoral stent graft for an inflammatory abdominal aortic aneurysm. Ann Vasc Surg 2006;20:408-10.

5. Copelan A, Chehab M, Cash C, Korman H, Dixit P. Endovascular management of ureteroarterial fistula: a rare potentially life threatening cause of hematuria. J Radiol Case Rep 2014;8:3745.

6. Coelho H, Freire, MJ, Azinhais P, Temido P. Arterioureteral fistula: an unusual clinical case. BMJ Case Rep 2016; bcr2016214400.

7. Yang $H$, Rios R, Lombardi J, Marella DM, Seftel AD. UreteroArterio-Enteric Fistula Formation and Stent Thrombosis After Endovascular Treatment of Ureteroarterial Fistula: A Case Report and Review of Literature. J Endourol Case Rep 2017;3:173-5.

8. Palmerola R, Westerman ME, Fakhoury M, Boorjian SA, Richstone L. Ureteroarterial Fistulas After Robotic and Open Radical Cystectomy. J Endourol Case Rep 2016;2:48-51.

9. Titomihelakis G, Feghali A, Nguyen T, Salvatore D, DiMuzio P, Abai $B$. Endovascular management and the risk of late failure in the treatment of ureteroarterial fistulas. J Vasc Surg Cases Innov Tech 2019;5:396-401.

10. Crane A, Rizzo A, Gong M, Sivalingam S. Ureteroarterial Fistula in a Patient with an lleal Conduit and Chronic Nephroureteral Catheter. J Endourol Case Rep 2019;5:64-7. 
11. Rittenberg L, Nordsiek M, Cahn D, Zhang K, Taylor N, Ginsberg P. Diagnosis and Management of a Challenging Patient: Ureteroarterial Fistula. Urology 2016;97:e9-e10.

12. Hashimoto M, Shimizu N, Toyoda S, Saito $Y$, Yamamoto $Y$, Minami $\mathrm{T}$, et al. Uretero-external iliac artery fistula with longterm indwelling of ureteral stent. Hinyokika Kiyo 2014;60:269-73.

13. Yamasaki T, Yagihashi $Y$, Shirahase T, Hashimura T, Okuno $\mathrm{T}$, Kudou $\mathrm{T}$, et al. Endovascular stent graft for management of ureteroarterial fistula: a case report. Hinyokika Kiyo 2004;50:641-4. 


\begin{tabular}{|c|c|c|c|c|c|c|c|c|}
\hline $\begin{array}{l}\text { Pacie } \\
\text { nte }\end{array}$ & $\begin{array}{l}\text { Ed } \\
\text { ad }\end{array}$ & $\begin{array}{l}\text { Se } \\
\text { xo }\end{array}$ & Factor de riesgo & Tipo de FUI & Clínica & Diagnóstico & Tratamiento & Complicación/mortalidad \\
\hline $1(3)$ & 54 & M & $\begin{array}{l}\text { Cancer de cérvix: CX } \\
+ \text { RT } \\
\text { Estenosis ureteral con } \\
\text { cambios de catéter } \\
\text { periódicos }\end{array}$ & Uréter - AIE I & $\begin{array}{l}\text { Sangrado por } \\
\text { nefrostomía }\end{array}$ & $\begin{array}{l}\text { Ureterografía/pielogra } \\
\text { fía }\end{array}$ & $\begin{array}{l}\text { Embolización de } \\
\text { All I+ } \\
\text { stent recubierto } \\
\text { Viabahn } 8 \text { x } 50 \\
\text { mm AIC I y } \\
\text { prox. I }\end{array}$ & No \\
\hline $2(4)$ & 77 & $\mathrm{H}$ & $\begin{array}{l}\text { Fibrosis } \\
\text { retroperitoneal por } \\
\text { AAA inflamatorio } \\
\text { operado mediante } \\
\text { endoprótesis } \\
\text { bifurcada } \\
\text { Estenosis ureteral con } \\
\text { cambios de catéter } \\
\text { periódicos }\end{array}$ & Uréter - AIC D & $\begin{array}{l}\text { Shock } \\
\text { hipovolémico }\end{array}$ & $\begin{array}{l}\text { Arteriografía } \\
\text { intraoperatoria }\end{array}$ & 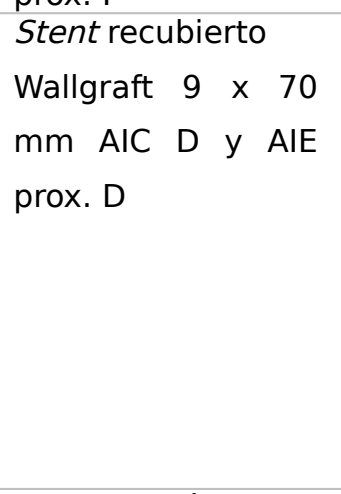 & No \\
\hline $3(5)$ & 70 & M & $\begin{array}{l}\text { - Cáncer de útero: CX } \\
+ \text { RT } \\
\text { - Portadora de } \\
\text { catéteres ureterales } \\
\text { por cálculos renales }\end{array}$ & Uréter - AIC D & $\begin{array}{l}\text { Hematuria } \\
\text { persistente }\end{array}$ & Arteriografía & $\begin{array}{l}\text { Embolización de } \\
\text { All D }+ \text { stent } \\
\text { recubierto Viabahn } \\
9 \times 5 \mathrm{~mm} \text { AIC D y } \\
\text { AlE prox. D }\end{array}$ & No \\
\hline $4(6)$ & 66 & $\mathrm{H}$ & $\begin{array}{l}\text { Infección de prótesis } \\
\text { aorto-bifemoral } \\
\text { (cirugía previa) }\end{array}$ & $\begin{array}{l}\text { Uréter - Rama I } \\
\text { de prótesis } \\
\text { aorto-femoral }\end{array}$ & $\begin{array}{l}\text { Hematuria } \\
\text { persistente } \\
\text { intraoperatori } \\
\text { a }\end{array}$ & $\begin{array}{l}\text { Ureterografía/pielogra } \\
\text { fía }\end{array}$ & $\begin{array}{l}\text { - Ureteroplastia } \\
\text { término-terminal } \\
\text { - Retirada de } \\
\text { prótesis vascular } \\
\text { infectada }\end{array}$ & $\begin{array}{l}\text { - Isquemia crítica Ell } \\
\text { - ASC EII }\end{array}$ \\
\hline
\end{tabular}




\begin{tabular}{|c|c|c|c|c|c|c|c|c|}
\hline $5(7)$ & 51 & M & $\begin{array}{l}\text { - Cáncer de cérvix: } \\
\text { CX + RT } \\
\text { - Estenosis con } \\
\text { catéteres ureterales } \\
\text { bilaterales }\end{array}$ & Uréter - AIE D & $\begin{array}{l}\text { Ureterorragia } \\
\text { durante } \\
\text { cambio de } \\
\text { catéter }\end{array}$ & $\begin{array}{l}\text { No se confirmó, alta } \\
\text { sospecha }\end{array}$ & $\begin{array}{l}\text { Stent recubierto } \\
\text { AIE D }\end{array}$ & $\begin{array}{l}\text { Oclusión del stent a los } 2 \\
\text { fístula urétero-arterio-entéri } \\
\text { laparotomía exploradora: retir } \\
\text { stent vascular + ligadura de } A \\
\text { ligadura de uréter D + res } \\
\text { segmentaria intestinal } \square \sin \text { is } \\
\text { posterior en la EID }\end{array}$ \\
\hline $6(8)$ & 82 & $\mathrm{H}$ & $\begin{array}{l}\text { - Cáncer vesical y } \\
\text { prostático con } \\
\text { cistoprostatectomía } \\
\text { tipo Bricker } \\
\text { - Portador de catéter } \\
\text { JJ por fuga en } \\
\text { anastomosis } \\
\text { ileoureteral }\end{array}$ & Uréter - AIC I & $\begin{array}{l}\text { Dolor en } \\
\text { flanco y } \\
\text { hematuria } \\
\text { masiva }\end{array}$ & Arteriografía & $\begin{array}{l}\text { Stent recubierto } \\
\text { Wallgraft } 12 \times 30 \\
\mathrm{~mm} \text { y } 12 \times 50 \mathrm{~mm} \\
\text { AIC I }\end{array}$ & No \\
\hline 7 (8) & 88 & $\mathrm{H}$ & $\begin{array}{l}\text { - Carcinoma vesical } \\
\text { con cistectomía tipo } \\
\text { Bricker } \\
\text { - Portador de catéter } \\
\text { por fuga en } \\
\text { anastomosis } \\
\text { ileoureteral izquierdo }\end{array}$ & Uréter - AIC I & $\begin{array}{l}\text { Hematuria } \\
\text { franca y } \\
\text { dolor en } \\
\text { ambos } \\
\text { flancos }\end{array}$ & Arteriografía & $\begin{array}{l}\text { Stent recubierto } \\
\text { Atrium } 10 \times 38 \mathrm{~mm} \\
\text { AIC I + stent no } \\
\text { recubiertos prox. y } \\
\text { distal }(14 \times 40 \mathrm{~mm} \\
\text { y } 12 \times 40 \mathrm{~mm})\end{array}$ & $\begin{array}{l}\text { Reintervención por persitencia } \\
\square \text { colocación de nuevo } \\
\text { recubierto Atrium } 10 \times 38 \mathrm{~mm} \\
\text { । }\end{array}$ \\
\hline $8(9)$ & 70 & M & $\begin{array}{l}\text { - Cáncer de cérvix: } \\
\text { CX + RT } \\
\text { - Estenosis con } \\
\text { catéter ureteral } \\
\text { bilateral }\end{array}$ & Uréter - AIE I & $\begin{array}{l}\text { Hematuria } \\
\text { durante } \\
\text { cambio de } \\
\text { catéter }\end{array}$ & Arteriografía & $\begin{array}{l}\text { Stent recubierto } \\
\text { Viabahn } 8 \times 50 \\
\text { mm AIE I }\end{array}$ & Sin seguimiento \\
\hline
\end{tabular}




\begin{tabular}{|c|c|c|c|c|c|c|c|c|}
\hline $9(9)$ & 77 & M & $\begin{array}{l}\text { - Cáncer de utero: CX } \\
+ \text { RT } \\
\text { - Estenosis con } \\
\text { catéter ureteral } \\
\text { bilateral. }\end{array}$ & Uréter - All D & $\begin{array}{l}\text { Hematuria } \\
\text { durante el } \\
\text { cambio de } \\
\text { catéter }\end{array}$ & Arteriografía & $\begin{array}{l}\text { Embolización All D } \\
+ \text { Extensión iliaca } \\
\text { (Gore) } 16 \text { x } 12 \text { x } \\
70 \text { mm en AIC y } \\
\text { AIE D }\end{array}$ & $\begin{array}{l}\text { Muerte al año por complicac } \\
\text { ERC progresiva }\end{array}$ \\
\hline $10(9)$ & 58 & M & $\begin{array}{l}\text { - Cáncer de cérvix: } \\
\text { CX + RT } \\
\text { - Estenosis con } \\
\text { catéter ureteral } \\
\text { bilateral }\end{array}$ & Uréter - All D & $\begin{array}{l}\text { Hematuria } \\
\text { franca }\end{array}$ & Arteriografía & $\begin{array}{l}\text { Stent recubierto } \\
\text { Atrium } 5 \times 22 \mathrm{~mm} \\
\text { en All D }\end{array}$ & No \\
\hline $11(9)$ & 58 & M & $\begin{array}{l}\text { - Cáncer de ovario y } \\
\text { recto: } \mathrm{CX}+\mathrm{RT} \\
\text { - Estenosis con } \\
\text { catéter ureteral } \\
\text { bilateral }\end{array}$ & Uréter - AIC D & $\begin{array}{l}\text { Hematuria } \\
\text { franca }\end{array}$ & $\begin{array}{l}\text { Ureterografía/pielogra } \\
\text { fía }\end{array}$ & $\begin{array}{l}\text { Stent recubierto } \\
\text { Viabahn } 9 \times 100 \\
\text { mm en AIC y AIE D }\end{array}$ & $\begin{array}{l}\text { Infección y oclusión del stent } \\
\text { con sangrado por nefrostomía } \\
11 \times 79 \mathrm{~mm} \text { en eje iliacc } \\
\text { antibioticoterapia crónica }\end{array}$ \\
\hline $12(9)$ & 56 & $\mathrm{H}$ & $\begin{array}{l}\text { Cáncer colorrectal: CX } \\
+ \text { RT }\end{array}$ & Uréter - All I & $\begin{array}{l}\text { Sangrado por } \\
\text { urostomía/ } \\
\text { ileostomía }\end{array}$ & Arteriografía & $\begin{array}{l}\text { Embolización All I } \\
+ \text { stent recubierto } \\
\text { eje iliaco I }\end{array}$ & $\begin{array}{l}\text { Infección de coils al de } 2 \\
\text { Amplatzer AIC I + ligadura } \\
\text { bypass fem } D \text { - fem I } \square \text { Sangrac } \\
\text { al de } 3 \text { meses } \square \text { exitus }\end{array}$ \\
\hline $\begin{array}{l}13 \\
(10)\end{array}$ & 64 & $\mathrm{H}$ & $\begin{array}{l}\text { - Cáncer vesical con } \\
\text { cistoprostatectomía } \\
\text { tipo Bricker } \\
\text { - Portador de catéter } \\
\text { J por estenosis en } \\
\text { anastomosis } \\
\text { ileoureteral izquierda }\end{array}$ & Uréter - AIC I & $\begin{array}{l}\text { Hematuria } \\
\text { intermitente } \\
+ \text { dolor en } \\
\text { flanco }\end{array}$ & Arteriografía & $\begin{array}{l}\text { Stent recubierto } \\
\text { LifeStream en AIC I }\end{array}$ & No \\
\hline
\end{tabular}




\begin{tabular}{|c|c|c|c|c|c|c|c|c|}
\hline $\begin{array}{l}14 \\
(11)\end{array}$ & 62 & $M$ & $\begin{array}{l}\text { - Cáncer de cérvix: } \\
\text { CX + RT } \\
\text { - } \\
\text { Tromboembolectomía } \\
\text { sobre AIE D } \\
\text { - Estenosis ureteral D } \\
\text { con catéter JJ }\end{array}$ & Uréter - AIE D & $\begin{array}{l}\text { Hematuria } \\
\text { franca } \\
\text { intermitente }\end{array}$ & $\begin{array}{l}\text { Ureterografía/pielogra } \\
\text { fía }\end{array}$ & $\begin{array}{l}\text { Stent recubierto } \\
\text { Viabahn en AIC y } \\
\text { AIE D }\end{array}$ & No \\
\hline $\begin{array}{l}15 \\
(12)\end{array}$ & 75 & $M$ & $\begin{array}{l}\text { - Cáncer de útero: CX } \\
+ \text { RT } \\
\text { - Hidronefrosis y } \\
\text { portadora de drenaje } \\
\text { JJ con recambios } \\
\text { periódicos }\end{array}$ & Uréter - AIE D & $\begin{array}{l}\text { Hematuria } \\
\text { aislada }\end{array}$ & TAC & $\begin{array}{l}\text { Stent recubierto } \\
\text { Fluency } 8 \times 60 \\
\text { mm en AIE D }\end{array}$ & No \\
\hline $\begin{array}{l}16 \\
(13)\end{array}$ & 76 & $\mathrm{H}$ & $\begin{array}{l}\text { - Cáncer vesical con } \\
\text { cistectomía tipo } \\
\text { Bricker } \\
\text { - Portador de catéter } \\
\text { JJ por estenosis en } \\
\text { anastomosis } \\
\text { ileoureteral izquierda }\end{array}$ & Uréter - AIC I & $\begin{array}{l}\text { Hematuria } \\
\text { masiva }\end{array}$ & $\begin{array}{l}\text { No se confirmó, alta } \\
\text { sospecha }\end{array}$ & $\begin{array}{l}\text { Stent recubierto } \\
\text { Passager en AIC y } \\
\text { AIE I }\end{array}$ & No \\
\hline 17 & 69 & $M$ & $\begin{array}{l}\text { - Cáncer de cérvix: CX } \\
\text { + RT } \\
\text { - Portadora de catéter } \\
\text { JJ por estenosis } \\
\text { ureteral derecha }\end{array}$ & Uréter - AIE D & $\begin{array}{l}\text { Hematuria } \\
\text { intermitente }\end{array}$ & Arteriografía & $\begin{array}{l}\text { Stent recubierto } \\
\text { Begraft } 8 \times 57 \mathrm{~mm} \\
\text { en AIE prox. D }\end{array}$ & $\begin{array}{l}\text { Pérdida progresiva de } \\
\text { hemodiálisis }\end{array}$ \\
\hline
\end{tabular}




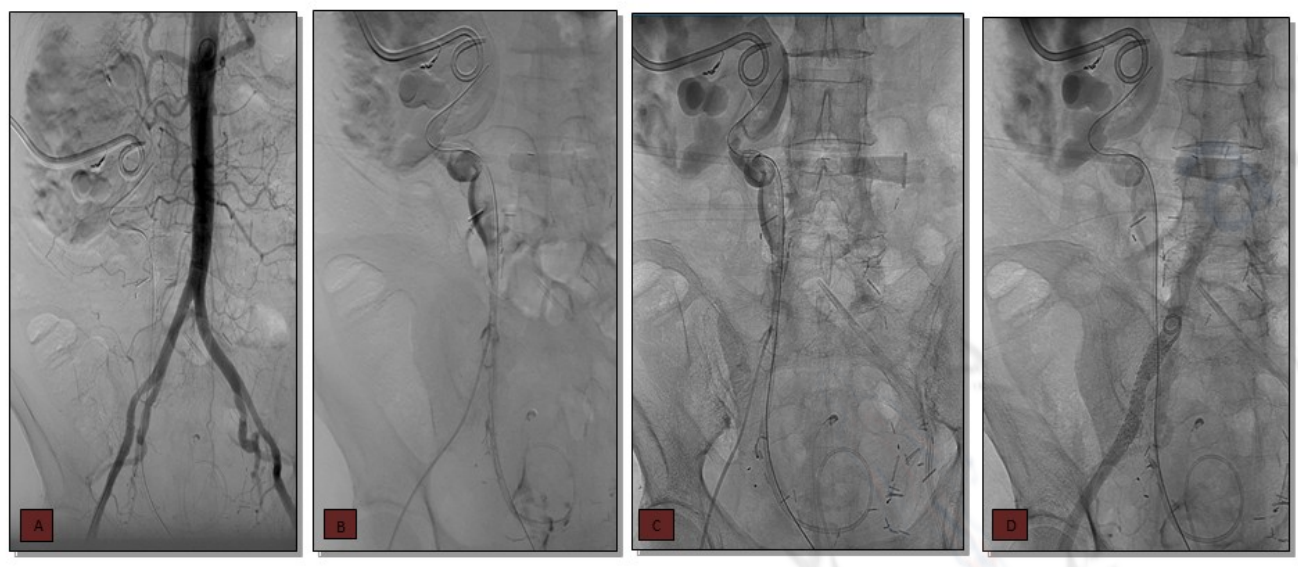

Figura 1. A. Aortografía para valorar la localización de las ramas principales renales e infrarrenales. B y C. Instilación de contraste y extravasación hacia el uréter, la pelvis renal y la vejiga. D. Implante de endoprótesis Begraft ${ }^{\circledR} 8 \times 57 \mathrm{~mm}$ en la arteria. 